\title{
Puisque commémoration il y a... Retour sur les études sandiennes à Grenoble
}

\section{Simone Vierne}

\section{(2) OpenEdition}

\section{Journals}

Édition électronique

URL : http://journals.openedition.org/recherchestravaux/167

DOI : 10.4000/recherchestravaux.167

ISSN : 1969-6434

Éditeur

UGA Éditions/Université Grenoble Alpes

\section{Édition imprimée}

Date de publication : 15 avril 2007

Pagination : 11-18

ISBN : 978-2-84310-107-7

ISSN : 0151-1874

\section{Référence électronique}

Simone Vierne, «Puisque commémoration il y a... Retour sur les études sandiennes à Grenoble », Recherches \& Travaux [En ligne], 70 | 2007, mis en ligne le 18 décembre 2013, consulté le 25 septembre 2020. URL : http://journals.openedition.org/recherchestravaux/167 ; DOI : https://doi.org/10.4000/ recherchestravaux.167 
Simone VIERNE

Université Stendhal-Grenoble 3

\section{Puisque commémoration il y a... Retour sur les études sandiennes à Grenoble}

Allocution prononcée en ouverture de la rencontre sur les Lettres d'un voyageur des I6 et I7 juin 2004, à Grenoble.

En cette année du bicentenaire de sa naissance, on voit enfin George Sand prendre sa véritable place dans le panthéon littéraire grâce à diverses manifestations - ce qui est bien plus judicieux que le projet heureusement avorté du transfert de ses cendres au Panthéon. Mais si la France adore les commémorations, les Français - et les spécialistes de littérature ne font pas exception sont, avec beaucoup d'ingratitude, oublieux de ceux qui n'ont pas attendu 2004 pour s'intéresser à George Sand autrement qu'en glosant sur ses aventures amoureuses. Au mieux, si on retenait alors quelque chose de son œuvre, c'étaient les "romans paysans", placés par les critiques et les éditeurs au rang de "bergeries» destinées à la jeunesse, longtemps les seuls accessibles. Car on n'a toujours pas d'édition complète des œuvres de George Sand, même si le projet est en route. Le reste de son œuvre romanesque était totalement oublié, et on la classait dans la littérature "idéaliste», comme en témoigne un court paragraphe dans Lagarde et Michard, où on la voit en compagnie d'Octave Feuillet et plus curieusement de Barbey d'Aurevilly! Voilà qui ne risquait pas de favoriser un regard critique valorisant de la communauté littéraire et universitaire. En outre, l'abondance de l'œuvre lui était comptée comme un défaut majeur, forcément féminin - car les femmes sont bavardes.

J'ai donc voulu, lors de ces journées consacrées aux Lettres d'un voyageur à l'université Stendhal de Grenoble, rendre hommage à ceux qui, il y a plus de trente ans, bravant l'opinion courante, ont formé un véritable groupe 
d'études sandiennes. Car il y fallait du courage et de l'originalité - cette dernière n'étant pas toujours bienvenue dans nos institutions universitaires, comme j'en ai fait l'expérience. Je pourrais d'ailleurs rattacher ce petit travail de mémoire aux Lettres d'un voyageur : de 1972 à 1974, elles étaient au programme de la seconde année de licence (on ne parlait pas encore de DEUG). Je m'en souviens bien car en l'enseignant durant ces deux années, je fus enfin en contact avec une œuvre autre que celles que j'avais lues ou étudiées, comme tout le monde, durant mon enfance et mes études au lycée, et que j'avais enseignées en cinquième ou quatrième. L'édition d'Henri Bonnet venait de sortir chez Garnier-Flammarion - c'est celle qui est rééditée actuellement - à l'instigation du professeur Léon Cellier qui avait détourné pour un temps Henri Bonnet de Gérard de Nerval. Faire publier les Lettres d'un voyageur dans une édition de ce qui deviendra le type même du «livre de poche" était, il faut l'avouer, une entreprise audacieuse : personne ne se souvenait de ce recueil, sauf de rares spécialistes du XIX siècle.

Je vais beaucoup parler de Léon Cellier. Pour commencer, je voudrais rappeler qu'en 1969, il avait dirigé et publié un Hommage à George Sand ${ }^{\text {, }}$ recueil de textes inédits, dont le Voyage en Auvergne et en Velay, et d'essais critiques, auquel beaucoup d'entre nous, ses anciens étudiants, ses assistants et ses collègues, participèrent. Pour l'anecdote, nous croyions, au moment de l'Hommage à George Sand, que George Sand avait pu passer ou même séjourner à Grenoble. Après tout, elle s'était rendue à Cognin, en Savoie, auprès de l'éditeur de La Revue des Deux Mondes, Buloz. Mais Georges Lubin, qui pouvait suivre presque heure par heure la vie de George Sand, à partir notamment de son édition de la Correspondance et de l'extraordinaire fichier qu'il avait constitué, nous prouva le contraire. En somme, qu'importe, d'autant que le lien de George Sand avec Grenoble, s'il n'est pas rattaché à sa présence réelle, existe bien : en I860, le peintre Diodore Rahoult et l'éditeur Dardelet publièrent une édition illustrée du poème, en patois du Dauphiné (en franco-provençal, dirions-nous maintenant), Le Grenoblo malherou, de Blanc la Goutte, écrit en 1773. Ils demandèrent une préface à George Sand, qui s'empressa de la fournir : comme on sait, elle encourageait tous les poètes ouvriers et paysans. Cela faisait partie de sa croyance profonde dans les vertus du peuple, y compris les vertus artistiques et créatrices.

Dans la présentation de cet Hommage, Léon Cellier notait :

Mais à vrai dire, cet hommage s'explique surtout par le fait qu'un heureux hasard a réuni à la faculté des Lettres de Grenoble trois professeurs qui avaient consacré

I. Hommage à George Sand, L. Cellier (dir.), Paris, PUF, 1969. 
leurs soins à publier des œuvres de George Sand. Jean Mallion que sa carrière avait conduit dans le Berry a édité pieusement les romans champêtres : La Mare au diable, François le Champi, La Petite Fadette, Les Maîtres sonneurs. Léon Guichard, musicologue éminent, s'est associé à Léon Cellier, adonné aux recherches occultes, pour exhumer le chef-d'œuvre méconnu que constitue une des sommes romanesques du romantisme, Consuelo et La Comtesse de Rudolstadt.

En outre, Léon Guichard, dans son ouvrage Le Romantisme et la Musique, célébrait George Sand en tant que musicienne, comme il le rappelle dans la présentation du colloque de 1974 dont je reparlerai. Léon Cellier était assurément un éminent dix-neuviémiste dont l'intérêt pour l'occultisme avait été à l'origine de sa thèse sur Fabre d'Olivet. Ses travaux, d'ailleurs, portaient sur des œuvres qui allaient bien au-delà du XIX ${ }^{e}$ siècle, de Claudel au cinéma. Mais il précise, dans cette présentation :

Pour moi, j'étais un sandiste en puissance. J'étais exaspéré par l'hostilité des disciples de Maurras à l'égard du romantisme en général et de George Sand en particulier. J'en voulais en particulier à Émile Henriot qui appelait George Sand "cette grosse femme à idées ${ }^{2}$ ».

Gêné par la sélection opérée dans son œuvre romanesque, il y voyait à juste titre l'effet «d'une sélection partisane, d'une manœuvre sournoise pour éliminer une certaine image de George Sand, la George Sand révoltée, la George Sand socialiste ${ }^{3}$. Il rappelle alors les propos d'Alain, qualifiant Consuelo d' " œuvre pascale» qui rend George Sand «immortelle».

C'est en I959 qu'avait eu lieu la publication, dans les Classiques Garnier, de Consuelo et de La Comtesse de Rudolstadt : trois volumes d'une édition critique exemplaire avec une très longue préface et des notes abondantes. Ces deux romans, qui n'en font qu'un, en fait, n'avaient jamais été réédités depuis le XIX ${ }^{e}$ siècle. Il faut ajouter qu'ils ne le furent pas non plus dans cette édition qui fait pourtant référence : en I976, l'éditeur fit en effet savoir à Léon Cellier qu'il n'envisageait pas une réédition du roman ${ }^{4}$. Il préférait évidemment rééditer La Mare au diable ou La Petite Fadette, qui se vendaient très bien pour Noël. Ce qui fait d'ailleurs que les éditions Garnier ne donnaient, prudemment, qu'un forfait au préfacier, comme me le racontait en souriant le professeur Jean Mallion.

2. La Porporina, Entretiens sur Consuelo, Actes du colloque de Grenoble, 4-5 octobre 1974, Grenoble, Presses universitaires de Grenoble, 1976, p. I.

3. Loc. cit.

4. Cette édition est finalement reprise, en cette année de bicentenaire, par Gallimard dans la collection «Folio", en 2 volumes, avec l'adjonction d'une postface de P. Laforgue. 
Il faut cependant rappeler que quelques universitaires avaient déjà eu l'originalité, car c'en était une, de jouer les pionniers dans le domaine des études sur George Sand : en particulier Pierre Salomon, dont la biographie de George Sand fut la première à s'intéresser enfin à l'œuvre et non à la seule vie de la romancière ; il avait donné, dès les années 30, une édition sélective des Lettres d'un voyageur's. Il faudrait aussi nommer Jean Pommier, qui montra ce que fut véritablement l'aventure de Venise et le rôle de George Sand dans la création de Lorenzaccio, et publia une intéressante et très neuve étude sur le «rêve monastique» chez George Sand, à propos du roman alors totalement oublié de Spiridion. Son élève Pierre Reboul étudia notamment les idées politiques et sociales de George Sand dans de nombreux articles et ouvrages entre I950 et 1979 .

Nous, les étudiants puis les assistants de Léon Cellier, Henri Bonnet, René Bourgeois, Huguette Burine, Béatrice Didier, Michelle Tourneur, nous étions naturellement amenés à nous intéresser, nous aussi, aux auteurs du XIXe siècle, et à George Sand en particulier. Mais nos directeurs de thèse, prudemment, nous confiaient d'autres sujets : une thèse, une "grande thèse" comme on les exigeait à l'époque, consacrée à George Sand, aurait été, dans les années 6o, fort mal vue des instances universitaires! Cependant, en 1974, l'université des Langues et Lettres, toujours anticonformiste, organisa un colloque que j'ai déjà mentionné, qui s'intitula «La Porporina, entretiens sur Consuelo». On y trouve les noms de Georges Lubin, Jean-Hervé Donnard, Jean-Pierre Lacassagne (le spécialiste de Pierre Leroux), Simone Vierne, René Bourgeois, Mireille Bossis, Jean-Marc Bailbé, Béatrice Didier. Il y avait aussi deux dix-huitiémistes, Jean Sgard et Michel Gilot (lequel éditera plus tard André, avec Huguette Burine, aux Éditions de l'Aurore) : n'oublions pas que Consuelo se passe au XVIII siècle.

Mais l'aventure la plus étonnante était à venir. On peut vraiment parler d'un "groupe de Grenoble d'études sandiennes». Ce groupe était tout à fait inédit et exceptionnel. Il aurait plu à George Sand, j'en suis certaine. En effet, il réunissait des éléments venus d'horizons très divers, et pas seulement universitaires. L'Association pour l'étude et la diffusion de l'œuvre de George Sand avait assurément un nom un peu compliqué, mais elle disait bien le problème qui se posait alors. Car l'œuvre était bien mal connue, et sa diffusion et son étude inexistantes en dehors des critiques très spécialisés que j’ai cités, et les rééditions très rares, en mettant à part, bien entendu, les « romans

5. G. Sand, Lettres d'un voyageur (extraits), édition de P. Salomon, Paris, Larousse « Classiques Larousse", s. d. [I935 selon le catalogue de la BNF], I vol. in-I6. 
paysans", limités à un public jeune. C'était une association de type loi de I90I, car il fallait bien lui donner une existence juridique. Mais ce qui était parfaitement original, c'est qu'elle unissait d'une part l'université Stendhal, d'autre part une ville limitrophe de Grenoble, Échirolles, à la population essentiellement ouvrière. L'intervention de cette dernière mérite quelque explication, car Échirolles pas plus que Grenoble n'étaient des villes où George Sand avait fait halte. Mais l'un de nos étudiants, Jean Courrier, qui travaillait sur George Sand, habitait cette commune et participait à la vie municipale, et l'un des adjoints au maire de l'époque, Alain Arvin-Bérod, s'enthousiasma à l'idée de mener un partenariat avec l'université et de lancer des opérations culturelles autour de George Sand. Cela d'autant plus qu'à Échirolles se trouvait, et se trouve encore, un centre compagnonnique, une "cayenne" des Compagnons du Devoir qui se destinent principalement aux métiers de la charpente et du bois. On sait quel rôle a joué George Sand dans la croisade que mena, au XIX ${ }^{e}$ siècle, Agricol Perdiguier pour réconcilier les Compagnons qui se battaient - très physiquement, en batailles rangées pour la suprématie de leurs Devoirs respectifs dans les villes où ils étaient embauchés. George Sand popularisa avec son roman Le Compagnon du Tour de France, en I840, les idéaux du compagnonnage, et aida matériellement Agricol Perdiguier. De sorte qu'il n'y a pas un musée du compagnonnage où l'on ne voie son portrait. Ainsi se trouvèrent unis des intérêts communs venus d'horizons très différents. La ville d'Échirolles nous offrit, outre des subsides que l'université n'avait déjà plus, sa bibliothèque, ses moyens de diffusion, ses locaux. Les Compagnons participèrent à toutes les manifestations. Les présidents successifs de l'université, Jean-Hervé Donnard, puis Jean Lavédrine, furent les présidents de cette association, et y consacrèrent beaucoup d'énergie. Jean-Hervé Donnard passa même de sa passion pour Balzac à un amour sans faille pour George Sand. Notre cher Jean Lavédrine, quoique angliciste, ne s'intéressa pas seulement à la gestion.

À partir de 1976 et jusqu'à 1990, l'Association a eu une grande et régulière activité. Elle publiait un bulletin, Présence de George Sand, revue semestrielle qui comportait des articles divers, souvent regroupés par thèmes : "George Sand et le compagnonnage», "George Sand et Rousseau", "Revenir aux Charmettes", "George Sand journaliste», "George Sand et la musique», "George Sand et Balzac», "George Sand et l'Italie», "George Sand et le théâtre", "George Sand et le XVIII siècle», "George Sand et Alexandre Dumas fils», "George Sand et l'autobiographie», "George Sand et la peinture», "La Russie et George Sand», "George Sand et l'Auvergne». Georges Lubin y a publié des lettres inédites, retrouvées après la publication d'un certain nombre de volumes de la Correspondance dans l'édition Garnier. Il avait 
aussi la gentillesse de répondre aux questions des lecteurs sur des points de la vie ou de l'œuvre de George Sand. La revue a publié des textes encore inédits ou introuvables: Thierry Bodin y a publié, d'après les manuscrits, Histoire du rêveur et La Prima Donna, qu'on ne connaissait que par la version incomplète et fautive d'Aurore Sand, L'Auberge rouge, Le Journal de Pagello, des Agendas (I866, I867, depuis publiés par Anne Chevereau), Les Charmettes, Fanchette. C'était, après les trois premiers numéros, encore artisanaux, une revue de belle facture, illustrée, dirigée par Jean Courrier, sur une maquette de Nicole Courrier. Sur le plan financier, cela ne pouvait se faire qu'avec le concours de la Mairie d'Échirolles. Il y a eu trente-six numéros.

L'Association a aussi organisé des colloques internationaux, car ils ne se limitaient pas à des participants français. Nous avons invité des sandiens italiens ou américains. Et tous n'étaient pas universitaires, même si évidemment l'université Stendhal fournissait la majorité des intervenants. Les Compagnons de la «cayenne» d'Échirolles, par exemple, assistaient et participaient à toutes les manifestations. Ils ont organisé l'une d'entre elles consacrée justement aux rapports entre George Sand et le Compagnonnage ; la salle des fêtes d'Échirolles était ornée de leurs outils et symboles, notamment de très belles cannes, et quelques chefs-d'œuvre de menuiserie - le portrait de George Sand trônant à la place d'honneur.

Je voudrais aussi rappeler que, si vous pouvez lire toute la Correspondance de George Sand dans une publication française, c'est parce que les membres du bureau de l'Association, sous la direction de Jean-Hervé Donnard, alors président de l'université Stendhal, sont intervenus auprès du ministre de la Culture de l'époque. Car les éditons Garnier avaient décidé, en 1976, d'arrêter la publication de la correspondance. Georges Lubin avait des propositions venues du Canada. Mais l'Association ne pouvait laisser partir à l'étranger, fût-ce au Québec, cette entreprise majeure, et son intervention fut efficace, car le ministère accorda, par l'entremise du Centre national des Lettres, une subvention qui adoucit les mœurs des éditeurs - qui avaient déjà, je le rappelle, refusé la réédition de Consuelo.

C'est aussi sous l'impulsion de l'Association que commença la publication des romans de George Sand, au départ prévue pour être complète, d'abord aux Presses universitaires de Grenoble (Jeanne et La Ville noire ${ }^{6}$ ) puis aux Éditions de l'Aurore, dans la collection dirigée par Jean Courrier. Cette

6. Il y aura trois rééditions de Jeanne, ce roman jusqu'alors totalement oublié, dont j’ai fait l'édition critique à la demande de Léon Cellier. La Ville noire fut éditée la même année par Jean Courrier. 
entreprise a perduré après la fin de l'Association, mais elle a elle aussi subi «les dures lois du marché». Et c'est bien dommage, car c'était une très belle édition pour la forme, et très sérieuse pour le fond : chaque roman était confié à un spécialiste et comportait une longue introduction, des notes, et sinon toutes les variantes, quand le manuscrit existait, du moins une étude de celles-ci. Elle était illustrée. C'est dans cette collection que mon ami René Bourgeois et moi-même avons publié, en 1983, une nouvelle édition de Consuelo et de La Comtesse de Rudolstadt, qui nous semblait un hommage nécessaire à notre maitre Léon Cellier. Elle a même eu une réédition, ce qui montre bien que malgré le prix et la forme (trois volumes, quand même...) on commençait à s'intéresser à George Sand en dehors de notre cercle grenoblois. Mais il a fallu attendre presque vingt ans pour avoir une édition chez Phébus, avec une simple préface de Gilles Lapouge, et 2004 pour l'édition beaucoup plus sérieuse de Damien Zanone et Nicole Savy dans la collection «Bouquins" des éditions Robert Laffont.

Notre travail a donc commencé à susciter un intérêt qui a dépassé le cadre local. Il faut dire qu'en 1976 s'étaient constituées les associations Friends of George Sand, aux États-Unis, avec laquelle j'ai été très vite en rapport, et Les amis de George Sand, d'abord basée à Lyon et qui songea un temps à fusionner avec notre groupe. Ces deux associations continuent de vivre avec beaucoup de vigueur, alors que la nôtre, hélas, s'est dissoute, survivant de peu à la disparition de la revue Présence de George Sand en 1990. Assurément, nous avons continué, René Bourgeois et moi, à diriger des thèses sur George Sand, écrire des articles, publier des romans. De même Béatrice Didier, avant beaucoup d'autres.

J'ai aussi organisé, en 1982, un colloque à Cerisy-la-Salle. Les communicants comprenaient, entre autres, certaines de nos amies sandiennes des Friends of George Sand, et Françoise van Rossum-Guyon, qui avait monté un groupe de recherches sandiennes à Amsterdam. Les dirigeants du Centre culturel de Cerisy n'étaient pas très optimistes quant au succès que pouvait rencontrer ce colloque et n'attendaient pas beaucoup de participants : ils furent heureusement surpris par leur nombre et leur qualité ! Clara Malraux, par exemple, fut une auditrice enthousiaste et active lors des discussions.

Ce qui précède n'est pas, croyez-le, un bilan nostalgique de type "anciens combattants». Après tout, nous sommes un certain nombre des "anciens» qui continuons à travailler sur l'œuvre. Mais d'une part, il m'a semblé juste, puisque les présentes Journées sont organisées par l'université de Grenoble, de rappeler quel rôle un certain nombre de Grenoblois et d'Échirollois avaient joué dans la "promotion" d'une œuvre alors très négligée, et de rendre hommage en particulier à Léon Cellier, qui fut à l'origine de ce mou- 
vement enthousiaste et un peu fou à l'époque. Le flambeau a été repris depuis, et les recherches sandiennes ont enfin en France un cadre quasi institutionnel avec le groupe d'études sandiennes organisé autour des universités Paris 7, Clermont-Ferrand 2 et Lyon 2. Damien Zanone relance les études sandiennes à Grenoble. Mais nous étions, et finalement j'en suis fière, un groupe pionnier, original par sa forme, sérieux par la qualité des recherches et des publications. Il m'a semblé nécessaire de rappeler aujourd'hui et dans cette université qu'il y a ici une tradition, et que si Grenoble n'a décidément pas eu l'honneur de voir George Sand un jour en ses murailles, elle a su lui rendre l'hommage que méritaient la femme et l'œuvre. 
Questions DE GenRe 